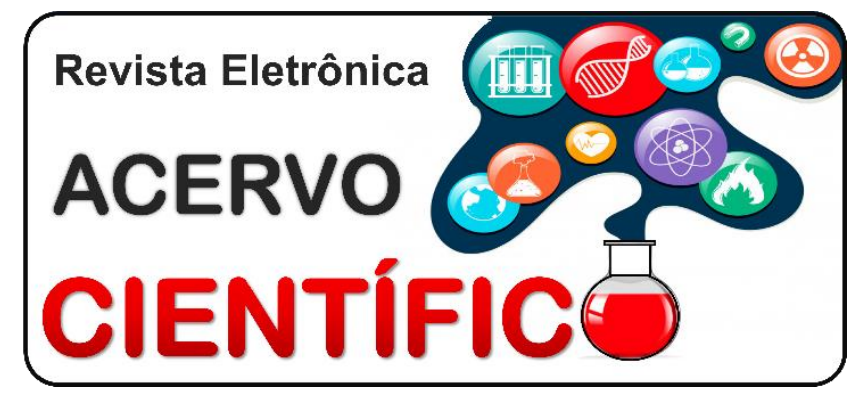

\section{ARTIGO ORIGINAL}

Recebido em: 10/2020

Aceito em: $11 / 2020$

Publicado em: 12/2020

\title{
Preocupação em relação à queda em idosos participantes de um progama de prevenção em um município do nordeste brasileiro
}

\author{
Concern about the fall in elderly participants in a prevention program in a northeast \\ brazilian municipality
}

\section{Preocupación por la caída de ancianos participantes en un programa de prevención en un municipio del noreste de brasil}

Felipe Lima Rebêlo ${ }^{1,2 *}$, José Raimundo Alves Neto ${ }^{1}$, Anna Beatriz Santos Florêncio ${ }^{2}$, Vitória Anjos da Silva ${ }^{2}$, Clarissa Cotrim dos Anjos Vasconcelos ${ }^{1}$,Thays Cristine Ferro Wanderley ${ }^{1}$, Aldir de Miranda Motta Neto ${ }^{1}$, Elaine Amado ${ }^{1}$.

\begin{abstract}
Resumo: Esse artigo buscou avaliar o medo de cair em um grupo de idosos participantes de um programa de prevenção de quedas em um município de Alagoas. Trata-se de um estudo transversal, realizado com os dados dos participantes do ambulatório de prevenção do risco de quedas em idosos em uma instituição hospitalar do nordeste brasileiro. Foram analisador os dados referentes ao medo de cair, através da escala Falls Eficacy Scale Internacional - Brasil (FES-I). Foi uma amostra composta por 51 indivíduos, onde identificou-se predominância do gênero feminino. A média de idade encontrada foi de 69,8 anos ( $d p= \pm 9,32$ ). As atividades que representzam maior medo foram: Limpar a casa, subir e descer escada, andar sobre superfície escorregadia, caminhar sobre superfície irregular, subir e descer ladeiras. Modelo multivariado que melhor explica a variação em FES-I foi boa percepção de saúde, escolaridade e repercussão funcional.As atividades que representam maior medo em relação a queda são as relacionadas ao ambiente que requer maior controle de equilíbrio para o desempenho das mesmas.
\end{abstract}

Palavras-chave: Idoso, Queda, Prevenção.

\begin{abstract}
This article sought to assess the fear of falling in a group of elderly people participating in a fall prevention program in a municipality in Alagoas. This is a cross-sectional study, carried out with data from participants in the outpatient clinic for the prevention of the risk of falls in the elderly in a hospital institution in northeastern Brazil. Data concerning fear of falling were analyzed using the Falls Eficacy Scale International Brazil (FES-I). It was a sample composed of 51 individuals, where a predominance of the female gender was identified. The average age found was 69.8 years $(s d= \pm 9.32)$. The activities that represent the greatest fear were: Cleaning the house, going up and down stairs, walking on a slippery surface, walking on an uneven surface, going up and down hills. Multivariate model that best explains the variation in FES-I was a good perception of health, education and functional repercussion. The activities that represent greater fear in relation to the fall are those related to the environment that requires greater balance control for their performance.
\end{abstract}

Keywords: Elderly, Fall, Prevention.

${ }^{1}$ Centro Universitário Cesmac, Maceió - AL.

*E-mail: feliperebelo_fisio@yahoo.com.br

2Universidade Estadual de Ciências da Saúde de Alagoas (UNCISAL), Maceió - AL. 
Resumen: Este artículo buscó evaluar el miedo a caer en un grupo de personas mayores que participan en un programa de prevención de caídas en un municipio de Alagoas. Se trata de un estudio transversal, realizado con datos de los participantes de la consulta externa para la prevención del riesgo de caídas en ancianos en una institución hospitalaria del noreste de Brasil. Los datos sobre el miedo a las caídas se analizaron mediante la Falls Eficacy Scale International - Brasil (FES-I). Se trató de una muestra compuesta por 51 individuos, donde se identificó un predominio del género femenino. La edad media encontrada fue de 69,8 años ( $\mathrm{dt}= \pm 9,32$ ). Las actividades que representan el mayor miedo fueron: Limpiar la casa, subir y bajar escaleras, caminar sobre una superficie resbaladiza, caminar sobre una superficie irregular, subir y bajar colinas. El modelo multivariado que mejor explica la variación en FES-I fue una buena percepción de salud, educación y repercusión funcional Las actividades que representan mayor miedo en relación a la caída son las relacionadas con el entorno que requiere mayor control del equilibrio para su desempeño.

Palabras clave: Anciano, Otoño, Prevención.

\section{INTRODUÇÃO}

A intensa transformação do padrão demográfico, nacionalmente e mundialmente, é uma das mais importantes modificações estruturais da sociedade moderna (INSTITUTO BRASILEIRO DE GEOGRAFIA E ESTATíSTICA - IBGE, 2011). O processo de envelhecimento é tema foco de diversas discussões, tanto em termos sociais quanto científicos, e a investigação das condições favoráveis a um envelhecimento com qualidade é tida como ponto primordial na melhoria da atenção à saúde da pessoa idosa (NASCIMENTO MM, 2019).

O processo de envelhecimento populacional ora referido, decorrente da transição demográfica, condicionou o processo de transição epidemiológica, levando a alterações importantes no quadro da morbimortalidade. A queda da mortalidade concentrou-se principalmente entre as doenças infecciosas. A tal fato atribui-se o desenvolvimento tecnológico na área de saúde, principalmente nas ações preventivas e controle de epidemias. Em contrapartida, esse contingente populacional passa a viver mais, e a conviver com fatores de risco para doenças crônico-degenerativas e à medida que envelhecem, tornam-se cada vez mais freqüentes as complicações dessas morbidades (MORLEY J, et al., 2019; CARMO JR, et al., 2020).

Diante desse quadro, o evento de queda se destaca entre as causas que mais afetam a saúde da pessoa idosa, podendo repercutir negativamente na vida do idoso gerando medo, preocupação e insegurança nestes indivíduos. O medo de cair pode desencadear uma postura de passividade em relação ao estado de saúde, levando muitas vezes a perda de autonomia e restrição as atividades diárias. Dessa forma é preciso atentar para esta variável quando se trata de promoção de saúde nesta população (CHINI LT, et al., 2019; BOCARDE L, et al., 2019).

Segundo o Ministério da Saúde (MS); cerca de 30\% das pessoas idosas caem a cada ano. Essa taxa aumenta para $40 \%$ entre os com mais de 80 anos e $50 \%$ entre os idosos institucionalizados. As mulheres tendem a cair mais que os homens até os 75 anos de idade, a partir dessa faixa etária as freqüências se igualam. Dos que caem, cerca de $25 \%$ requerem hospitalização e, destes, apenas metade sobreviverá após um ano (MINISTÉRIO DA SAÚDE, 2006). A literatura relata Fernandes M, et al. (2009), que o medo de cair pode acontecer entre $12 \%$ e $65 \%$ dos idosos com mais de 60 anos que vivem independentes na comunidade. Para aqueles com mais de 60 anos que caíram, o medo varia de $29 \%$ e $92 \%$.

O objetivo deste trabalho foi avaliar o medo de cair em um grupo de idosos participantes de um programa de prevenção de quedas em um município de Alagoas.

\section{MÉTODOS}

Este estudo trata-se de uma pesquisa observacional com abordagem quantitativa, descritiva e analítica realizada em um município de Alagoas. Este artigo é um braço de pesquisa do Projeto guarda-chuva intitulado Avaliação multidimensional da eficácia de um programa de prevenção de quedas em idosos: um projeto 
guarda-chuva. O protocolo da pesquisa teve projeto aprovado pelo Comitê de Ética e Pesquisa da Centro Universitário Cesmac sob o protocolo de número 1669-12.

A coleta de dados foi realizada com os dados dos pacientes vinculados ao ambulatório de prevenção do risco de quedas em idosos de uma Instituição Hospitalar do Nordeste Brasileiro. As informações coletadas foram registradas em uma ficha de recolhimento de dados previamente elaborada para este fim. Para esta pesquisa foram incluídos todos os pacientes, sendo as avaliações desde maio de 2011 a novembro de 2012.

O ambulatório de prevenção do risco de quedas é um projeto de educação e promoção em saúde vinculado ao Serviço de Geriatria e Gerontologia da instituição hospitalar onde teve suas atividades iniciadas em maio de 2011. As atividades acontecem nas dependências da Unidade Docente Assistencial Professor Rodrigo Ramalho, no espaço físico onde funciona o ambulatório de Fisioterapia motora.

Esse projeto acontece todas as segundas feiras, no período vespertino, durante 4 meses, com intervensões grupais de 8 pessoas e duração de 2 horas. Para participar no projeto os indivíduos devem ter idade igual ou superior a 50 anos e não necessitam possuír risco de quedas. Os pacientes são encaminhados por médicos, profissionais da área da saúde ou podem ainda se inscreverem por conta própria.

Primeiro os participantes são convidados à uma sala de aula assistir conteúdo teórico inicialmente sobre prevenção de quedas, havendo trocas de temas a cada semana, ministrados pelos profissionais da equipe interdisciplinar de Geriatria e Gerontologia (médico geriatra, fisioterapeuta, psicólogo, nutricionista, assistente social, enfermeiro, educador físico, terapeuta ocupacional e fonoaudiólogo).

Todos os participantes passam por uma avaliação multidimensional no início das atividades do projeto, onde são colhidos todos os dados sócio demográficos e econômicos e avaliam-se: percepção de saúde, estado cognitiva, de humor, qualidade de vida, capacidade funcional, equilíbrio e mobilidade e medo de quedas. A mesma avaliação é repetida ao término das atividades. Para este artigo, realizou-se uma abordagem transversal dos dados da avaliação inicial do projeto e foram analisados os dados socioeconômicos e demográficos e as questões relacionadas ao medo de queda, referentes a escala Falls Efficacy Scale Internacional - Brasil.

A escala Falls Efficacy Scale Internacional - Brasil (FES-I-BRASIL) utilizada é uma versão adaptada e validada por Camargos FF, et al. (2010). Ela tem por objetivo avaliar o medo de cair em 16 atividades diárias. A pontuação final é interpretada de forma crescente como indivíduos que não tem preocupações em cair, aqueles que atingem menores pontuações, ou indivíduos com muita preocupação em cair, aqueles que atingem maiores pontuações. São avaliadas atividades de vida diária, usualmente desempenhadas no dia a dia do indivíduo, como limpar a casa, tomar banho, subir e descer escadas, sair para visitar parentes, entre outras.

Para elucidar a influência das diversas variáveis socioeconômicas sobre os escores do teste FES-I realizou-se uma regressão linear multivariada. Para tal, primeiro os escores de FES-I foram transformados logaritmicamente para atenderem ao pressuposto da normalidade. Após isso, uma regressão linear multivariada, com modo de entrada passo a passo, foi conduzida para identificar o modelo que melhor explicasse a variação nos escores de FES-I. A confecção do banco de dados foi realizada no Programa Excel ${ }^{\circledR} 2003$ (ambiente Windows $\AA$ ), sendo a análise destes realizada no pacote estatístico SPSS $\AA^{(S t a t i s t i c a l ~}$ Package for Social Sciences) versão 15.0.

\section{RESULTADOS}

A amostra desse estudo foi composta por 51 indivíduos, onde identificou-se predominância do gênero feminino (82\%). A média de idade encontrada foi de 69,8 anos ( $\mathrm{dp}= \pm 9,32$ ), sendo a mínima de 59 anos e a máxima 89 anos. A maioria dos sujeitos avaliados apresentou alta escolaridade> 9 anos (49\%), sendo a média de estudo de 9,25 anos ( $d p= \pm 5,98)$, com renda $>1$ salário (51\%) e $58 \%$ relataram não participação em grupos de idosos. No que se diz respeitos aos dados relacionados ao estado de saúde, a população estudada apresentou uma média 2,52 doenças ( $\mathrm{dp}= \pm 0,7$ ), possuindo um hitórico de $\geq 3$ doenças $(64,7 \%)$. Quanto as medicações a maioria relatou o uso de até 3 medicações $(41,1 \%)$, que não praticam atividades físicas $(54,9 \%)$. 
Relataram também alto uso de lentes corretivas $(68,6 \%)$ negaram uso de próteses auditivas $(90,2 \%)$, e avaliaram sua saúde como boa $(74,5 \%)$.

No que concerne aos dados relacionadas ao número de quedas, a maior parte dos indivíduos relatou ter sofrido uma no ultimo ano (66,6\%), com média de 1,47 quedas ( $\mathrm{dp}= \pm 1,75)$. A maioria, $25(49 \%)$, atribuiu como causa desta um tropeção ou escorregão e $41(80,4 \%)$ negaram sofrer repercursão funcional após o evento.

No Gráfico 1 estão distribuídos os resultados por atividades na FES- I, onde as atividades que representaram maior medo foram: limpar a casa, subir ou descer escadas, andar sobre superfície escorregadia, caminhar sobre superfície irregular e subir ou descer ladeira.

Gráfico 1 - Distribuição dos resultados por atividades na FES-I.

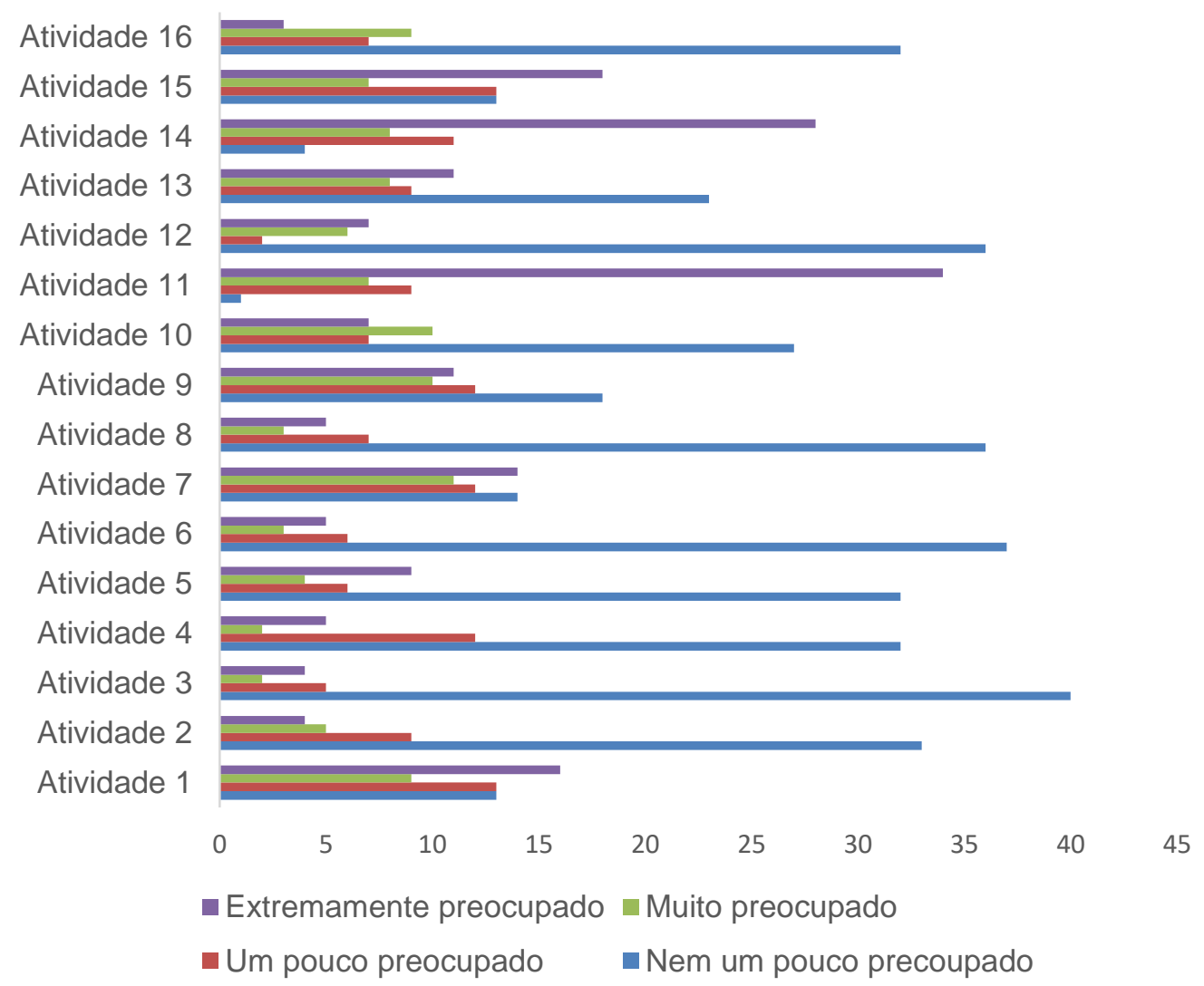

Fonte: REBÊLO FL, et al., 2020.

Na Tabela 1 estão dispostos os resultados da análise de regressão linear multivariada, onde verificou-se que o modelo constituído pelo estado de saúde atual dos indivíduos, pelos anos de escolaridade e pela presença de repercussão funcional pós queda foi o que melhor explicou a variação dos valores absolutos de FES-I ( $R^{2}$ Ajust. $=0,36$; Durbin-Watson $\left.=1,6 ; F=10,3 ; p<0,001\right)$.

Tabela 1 - Modelo multivariado que melhor explica a variação em FES-I.

\begin{tabular}{ccccccc}
\hline \multirow{2}{*}{ Modelo } & \multicolumn{2}{c}{ Coeficientes } & \multirow{2}{*}{$\mathbf{P}$} & \multicolumn{2}{c}{ IC95\% } \\
\cline { 2 - 3 } \cline { 6 - 7 } & B & Erro Padrãon & & & Limite Inferior & Limite Superior \\
\hline (Constante) & 4,08 & 0,169 & $<0,01$ & & 3,743 & 4,422 \\
Boa percepção de saúde & $-0,29$ & 0,09 & $<0,01$ & & $-0,477$ & $-0,113$ \\
Escolaridade & $-0,01$ & 0,007 & 0,016 & & $-0,030$ & $-0,003$ \\
Repercussão funcional & 0,23 & 0,09 & 0,020 & & 0,038 & 0,434 \\
\hline
\end{tabular}

Fonte: REBÉLO FL, et al., 2020. 


\section{DISCUSSÃO}

As características sócio demográficas e econômicas da população estudada refletem a realidade dos idosos brasileiros, onde evidenciou-se uma população predominantemente feminina, casados e que vivem com uma renda de um salário mínimo (IBGE, 2009).

Quanto a escolaridade, identificou-se uma particularidade, onde, diferentemente da maioria dos idosos brasileiros, e residentes em Alagoas, verificou-se uma grande proporção de participantes com alta escolaridade, divergindo da maioria dos estudos com população semelhante (MAZO GZ, et al., 2007). A predominância de idosos com alta escolaridade pode ser explicada pela maior procura por programas de saúde pelos indivíduos com maior esclarecimento. A escolaridade é tida como um fator de proteção à saúde, visto que indivíduos com maior grau de instrução tendem a se preocupar mais com esse constructo (SOUZA JN e CHAVES EC, 2005).

Já sobre a presença de alterações auditivas e visuais que é considerada comum em idosos, e nem sempre recebe a devida atenção, sendo um problema que pode acarretar em diminuição da capacidade funcional, propiciando um risco maior de quedas (NUNES MCR, et al., 2009). No presente estudo, identificou-se um alta proporção de sujeitos que relataram alterações visuais e uso de lentes corretivas.

Todos os aspectos acima discutidos podem interferir diretamente na percepção de saúde do indivíduo. A auto avaliação da saúde em indivíduos com faixas etárias mais avançadas vem sendo considerada um fator primordial na saúde do idoso. Apesar de ter uma íntima relação com a presença de enfermidades, a subjetividade desse constructo vai além da presença de doenças, e parece estar mais relacionada a implicação funcional do que necessariamente a presença da co-morbidade (FHON JRS, et al., 2012).

Apesar de não restringir a faixa etária para participação no projeto, a maioria dos indivíduos participantes são idosos. A predominância de indivíduos nessa faixa etária pode justificar a maior proporção de sujeitos com três ou mais co-morbidades bem como aqueles que fazem uso contínuo de mais de três medicações, fato esse corroborado pelo estudo de (GUIMARÃES LH, 2009).

O envelhecimento leva uma diminuição da reserva funcional, o que predispõe o idoso ao desenvolvimento de co-morbidades, e consequentemente aumenta a necessidade do uso de fármacos (ARAÚJO MF, et al., 2018). Resultados semelhantes foram encontrados no estudo de Barros SL, et al. (2012), ressaltando que a polifarmácia destaca-se como uma das causas de quedas em indivíduos de faixa etária mais avançada, e o uso de medicamentos vem sendo associado pelo risco de fraturas pós queda nessa população, principalmente os que tem como consequência sonolência e alteração de equilíbrio.

Dessa forma percebe-se que a população de idosos tende a apresentar maiores problemas de saúde que os demais grupos etários, o que pode interferir negativamente na capacidade funcional desses idosos (SANTOS SCA e FIGUEIREDO DMP, 2019). Dentro desse contexto, destaca-se que culturalmente esses indivíduos, em geral, não tem o hábito da prática de atividade física, fato evidenciado nesse estudo, onde a maioria dos idosos relatou sedentarismo, segundo Siqueira FV, et al. (2007) em estudo com idosos da comunidade que objetivou identificar fatores de risco associado a quedas também encontraram uma alta prevalência de sedentarismo, onde $76,5 \%$ da amostra relatou não realizar nenhum tipo de atividade física.

Em análise aos dados deste estudo, verificou-se que mais da metade dos sujeitos relataram pelo menos uma queda no último ano, dado este corroborado por Lopes KT, et al. (2009) que encontrou um histórico positivo de queda em mais de $80 \%$ da população estudada. Esse dado pode ser explicado pelo fato de ser um projeto que recebe também indivíduos com risco de quedas e pela faixa etária dos indivíduos, em quase sua totalidade formada por idosos (FIEDLER MM e PERES KG, 2008).

Em conformidade aos dados da literatura Nachreiner NM, et al. (2007) nesse estudo, a maioria dos indivíduos que relataram quedas caíram no ambiente domiciliar, o que pode ser explicado pela familiaridade do local, que representaria maior segurança. No entanto, na maioria das vezes o domicílio pode se tornar um local de risco, já que o desempenho das atividades nesse ambiente, geralmente são realizadas com menor grau de atenção, devido a autoconfiança ora relatada (SILVA TM, et al., 2007). 
A avaliação do medo de cair pela FES-I, revelou grande receio à quedas nas atividades de limpar a casa, subir ou descer escadas, andar em superfície escorregadia, caminhar sobre superfície irregular e subir ou descer uma ladeira. Esses dados são extremamente corroborados por Lopes KT, et al. (2009) onde, com exceção da atividade de limpar a casa, todas as outras estavam entre as de maior receio em relação a queda para a população estudada. A análise da regressão linear multivariada revelou associação inversa entre boa percepção da saúde e escolaridade com a FES-I, ou seja perceber a saúde como boa e ter maior escolaridade reduz os valores no instrumento, o que representaria que estes dois fatores atuam como protetores para 0 medo de cair.

A boa percepção de saúde pode está diretamente relacionada a uma postura positiva em relação ao componente físico e psicológico, onde indivíduos que avaliam esse constructo como ruim tendem a ter maiores problemas de saúde, além de apresentarem tendência a maior comprometimento funcional, o que poderia aumentar a probabilidade de apresentar distúrbios de equilíbrio e consequentemente, maior risco de quedas, implicando em maior receio em cair durante o desempenho das atividades diárias (SMITH AA, et al., 2017).

A escolaridade também mostrou significância estatística quando relacionada a FES-I. indivíduos com maior nível educacional, tendem a apresentar melhores padrões de saúde e maior esclarecimento, o que poderia justificar tanto uma melhor percepção quanto melhores padrões de saúde , levando a adoção de uma postura mais positiva frente ao desempenho das atividades diárias (ABREU DR, et al., 2016).

Wilson RT, et al. (2006) em estudo de coorte, acompanhou durantes dois anos um grupo de 5630 idosos e identificou relação positiva entre a baixa escolaridade e o risco para a ocorrência de fratura de quadril em idosos da comunidade.

A repercussão funcional pós queda apresentou associação positiva com a FES-I, o que representa que ter tido algum tipo de repercussão aumenta os valores na escala. Após um evento de queda, boa parte dos idosos podem desencadear alterações psicológicas como medo e ansiedade e insegurança. Esses sentimentos, tendem a levar o indivíduo a isolar-se socialmente devido ao medo de locomover-se pelo receio de um novo evento (BARBOSA MD, et al., 2017).

A avaliação do medo de cair em idosos pode fornecer informações importantes acerca do receio e do estilo de vida do indivíduo no que diz respeito ao evento de queda. Para os indivíduos que já sofreram queda, e principalmente para os que cursaram com alguma repercussão funcional, as limitações impostas por este processo pode gerar desde insatisfações até restrição total para o desempenho de determinadas atividades de vida diária. Uma vez que o idoso perceba um risco eminente ou desenvolva um trauma pós queda, o mesmo pode assumir uma postura de passividade frente as atividades cotidianas, e este fator comportamental pode acelerar o declínio funcional.

Dessa forma, sabe-se que o medo de cair trás consequências negativas no âmbito físico e social, mas ele não pode se tornar um fator determinante para não realização das atividades, já que, essa postura passiva, pode condicionar a inatividade física, o que poderia representar menor estímulo aos sistemas responsáveis pela manutenção do equilíbrio, o que aceleraria o declínio fisiológico, e por fim configuraria em maior risco de quedas (VIEIRA GC, et al., 2019).

\section{CONCLUSÃO}

O presente estudo mostra que as quedas ocorrem pela combinação de vários fatores. As atividades que representaram maior medo em relação a queda foram as relacionadas a ambientes com superfícies instáveis, como pisos escorregadios e irregulares, o que representaria uma necessidade de maior controle de equilíbrio para o desempenho das mesmas.A escolaridade a boa percepção de saúde mostraram-se como fatores protetores para o medo de queda, ao passo que ter repercussão funcional após o evento de queda, mostrou como fator de risco para um maior receio a um novo evento. 


\section{REFERÊNCIAS}

1. ABREU DR, et al. Factors associated with recurrent falls in a cohort of older adults. Revista Ciencia e Saude Coletiva, $2014 ; 21(11)$ : 3439-3446.

2. ARAÚJO MF, et al. Record of the circumstances of falls in the community: Perspective in the Iberian Peninsula. Revista Latino-Americana de Enfermagem, 2018.

3. BARBOSA MD, et al. Caiu, tchau e bença: os sentimentos, comportamentos e soluções de idosos após a queda em residências unipessoais - If you fell, bye and God bless: feelings, behaviour and the found solutions after a fall at home. RECIIS, 2017; 11(3): 1-16.

4. BARROS SL, et al. Correlação entre inatividade física, polifarmácia e quedas em idosos. ConScientiae Saúde, 2012; 11(1): 19-29.

5. BOCARDE L, et al. Medo de quedas e força muscular do quadril em idosos independentes da comunidade. Fisioterapia e Pesquisa, 2019; 26(3): 298-303.

6. CAMARGOS FF, et al. Adaptação transcultural e avaliação das propriedades psicométricas da Falls Efficacy Scale International em idosos brasileiros (FES-I-BRASIL). Revista Brasileira de Fisioterapia, 2010;14(3): 237-243.

7. CARMO JR, et al. Falls of Patients With Home Care: Prevalence and Associated Factors. Reme Revista Mineira de Enfermagem, 2020; 24(2): 1-10.

8. CHINI LT, et al. Validation of the fall risk tracking tool (FRRISque) in elderly community dwellers. Ciencia e Saude Coletiva, 2019; 24(8): 2845-2858.

9. FERNANDES $M$, et al. A influência da fisioterapia na prevenção de quedas em idosos na comunidade: estudo comparativo. Motriz, 2009; 15(3): 527-536.

10. FHON JRS, et al. Quedas em idosos e sua relação com a capacidade funcional. Rev Lat Am Enfermagem, 2012; 20(5): 927-34.

11. FIEDLER MM, PERES KG. Functional status and associated factors among the elderly in a southern Brazilian city: a population-based study. Cad Saude Publica, 2008;24(2):409-15.

12. GUIMARÃES LH, et al. Avaliação da capacidadefuncional de idosos em tratamento fisioterapêutico. Revista Neurociências, 2009; 12(3).

13. INSTITUTO BRASILEIRO DE GEOGRAFIA E ESTATÍSTICA - IBGE. De Geografia E Estatística - Ibge. Rio de Janeiro, 2009;39(3).

14. LOPES KT, et al. Prevalência do medo de cair em uma população de idosos da comunidade e sua correlação com mobilidade,equilíbrio dinâmico,risco e histórico de quedas.Rev Bras Fisioter, 2009;13(3):223-9.

15. MAZO GZ, et al. Condições de saúde, incidêcia de quedas e nível de atividade física dos idosos. Rev. bras. fisioter, 2007; 11(6).

16. MORLEY J, et al. Controle postural estático e risco de quedas em mulheres idosas com e sem incontinência urinária. Fisioterapia e Pesquisa, 2019; 26(1): 1-11.

17. NACHREINER NM, et al. Circumstances and consequences of falls in community-dwelling older women. J. Womes Health,2007; 16 (10):1437-46.

18. NASCIMENTO MM, Queda em adultos idosos: considerações sobre a regulação do equilíbrio, estratégias posturais e exercício físico. Geriatrics, Gerontology and Aging, 2019; 13(2):103-110.

19. NUNES MCR,et al. Influência das características sócio demográficas e epidemiológicas na capacidade funcional de idosos residentes em Ubá. Minas Gerais.Rev Bras Fisioter. 2009;13(5): 376-82.

20. SANTOS SCA, FIGUEIREDO DMP. Predictors of the fear of falling among community-dwelling elderly Portuguese people: An exploratory study.Ciencia e Saude Coletiva, 2019;24(1): 77-86.

21. SILVA TM, et al. A vulnerabilidade do idoso para as quedas: análise dos incidentes críticos. Revista Eletrônica de Enfermagem, 2007; 9(1): 64-78.

22. SIQUEIRA FV, et al. Prevalência de quedas em idosos e fatores associados. Rev Saúde Pública, 2007;41(5): 74956.

23. SMITH AA, et al. Avaliação do risco de quedas em idosos residentes em domicílio. Revista Latino-Americana de Enfermagem, 2017; 25(1).

24. SOUZA JN,CHAVES EC. O efeito do exercício de estimulação da memória em idosos saudáveis. Rev Esc Enferm USP, 2005; 39(1):13-9.

25. VIEIRA GC, et al. Avaliação do medo de cair e da velocidade da marcha em idosos residentes em uma instituição de longa permanência: relato de experiência. HU Revista 2019,45(2): 227-230.

26. WILSON RT, et al. Hip fracture risk among community-dwelling elderly people in the United States: a prospective study of physical, cognitive and socioeconomic indicators. Am j Publ Health. 2006; 96:1210-8. 